\title{
Correction to: Language, Social Media and Ideologies
}

\section{Correction to:}

\section{S. Dovchin, Language, Social Media}

\section{and Ideologies, SpringerBriefs in Linguistics,}

https://doi.org/10.1007/978-3-030-26139-9

The original version of the book was inadvertently published without following consistency in the chapter end references, which have now been corrected. The chapter has been updated with the change. 